INPLASY

PROTOCOL

To cite: Zhu et al. Evaluation of contrast-enhanced ultrasound for activity of rheumatoid arthritis: A protocol for systematic review and metaanalysis. Inplasy protocol 2020120125. doi: 10.37766/inplasy2020.12.0125

Received: 24 December 2020

Published: 25 December 2020

Corresponding author: Hui Wang

Indlnsd@163.com

Author Affiliation:

Ultrasound department of the First Affiliated Hospital of

Dalian Medical University

Support: Liaoning Natural Science Found.

Review Stage at time of this submission: Data extraction.

Conflicts of interest:

None.

\section{Evaluation of contrast-enhanced ultrasound for activity of rheumatoid arthritis: A protocol for systematic review and meta-analysis}

Zhu, Y1; Sui, P2; Wang, C3; Wang, $\mathrm{H}^{4}$.

Review question / Objective: Explore the value of CEUS in the activity of RA disease.

Condition being studied: Contrast-enhanced ultrasound (CEUS) refers to a technique that uses contrast medium to strengthen the echo of backscatter, which can significantly improve the resolution, sensitivity and specificity of ultrasound diagnosis. As a quantitative imaging examination of blood flow signals, CEUS has allowed detection of synovial microvascularization in the joints of patients with rheumatoid arthritis (RA). Therefore, the purpose of this study is to evaluate the value of CEUS in the activity of RA disease.

INPLASY registration number: This protocol was registered with the International Platform of Registered Systematic Review and Meta-Analysis Protocols (INPLASY) on 25 December 2020 and was last updated on 25 December 2020 (registration number INPLASY2020120125).

\section{INTRODUCTION}

Review question / Objective: Explore the value of CEUS in the activity of RA disease.

Condition being studied: Contrastenhanced ultrasound (CEUS) refers to a technique that uses contrast medium to strengthen the echo of backscatter, which can significantly improve the resolution, sensitivity and specificity of ultrasound diagnosis. As a quantitative imaging examination of blood flow signals, CEUS has allowed detection of synovial 
microvascularization in the joints of patients with rheumatoid arthritis (RA). Therefore, the purpose of this study is to evaluate the value of CEUS in the activity of RA disease.

\section{METHODS}

Search strategy: We will search Pubmed as follows: rheumatoid arhritis OR RA[Title/ Abstract] AND contrast-enhanced ultrasound $O R$ contrast-enhanced ultrasonography OR contrast-enhanced ultrasonic diagnosis OR contrast-enhanced sonography OR contrast-enhanced echography OR contrast-enhanced echotomography OR CEUS[Title/Abstract]. The similiar search strategy will also used to other database.

Participant or population: All patients were in accordance with diagnostic criteria of RA established by the American College of Rheumatology (ACR) in 1987 or the European alliance against Rheumatism (EULAR)/ACR in 2009 or the Chinese institute of Rheumatology in 2010 or the World Health Organization (WHO)in 2008 in spite of race, nationality, and sex.

Intervention: All patients were assessed with CEUS and Laboratory examination.

Comparator: All patients were assessed with CEUS and Laboratory examination.

Study designs to be included: This study will only include high quality clinical cohort or case control studies.

Eligibility criteria: Participants: All patients were in accordance with diagnostic criteria of RA established by the American College of Rheumatology (ACR) in 1987 or the European alliance against Rheumatism (EULAR)/ACR in 2009 or the Chinese institute of Rheumatology in 2010 or the World Health Organization (WHO)in 2008 in spite of race, nationality, and sex. Intervention and comparison: All patients were assessed with CEUS and Laboratory examination. Outcomes: The primary outcomes include a quantitative scoring system, through which synovial vascularity intensity was evaluated by means of CEUS. Type of study: This study will only include high quality clinical cohort or case control studies.

Information sources: We will search PubMed, Embase, Cochrane Library, and CNKI from their inception to the December 20, 2020, without restrictions of language and publication status.

Main outcome(s): The primary outcomes include a quantitative scoring system, through which synovial vascularity intensity was evaluated by means of CEUS.

Quality assessment / Risk of bias analysis: The methodological quality was independently assessed by 2 investigators according to the methodological index for nonrandomized studies(MINORS).The MINORS criteria included 12 assessment items and each of these items was scored as"yes"(2), "no"(0), or"unclear".MINORS score ranged from 0 to 24 ; and score $\geq 13$ indicated a good quality. Any disagreements between two investigators will be solved through discussion or consultation by a third investigator.

Strategy of data synthesis: The following data were extracted from the eligible studies: year of article, the first author's surname, sample size, examination position, mean of disease activity score of 28 joints, Instrument.

Subgroup analysis: Experimental group will be included in each group.

Sensibility analysis: The Cochrane Qstatistic and 12 test were used to evaluate potential heterogeneity between studies. If $Q$ test shows a $\mathrm{P} 50 \%$ which indicates heterogeneity, the random-effect model was conducted, or else the fixed-effects model was used.

Language: Without restrictions.

Country(ies) involved: China. 
Keywords: rheumatoid arthritis; metaanalysis; contrast-enhanced ultrasound.

Contributions of each author:

Author 1 - Yunpei Zhu - The author searched the database, extracted data, wring the original draft and review.

Email: 445607833@qq.com

Author 2 - Ping Sui - The author searched the database ,extracted data, wring the original draft and review.

Email: 1558397201@qq.com

Author 3 - Cong Wang - The author contributed to the conceptualization.

Email: wc027214@163.com

Author 4 - Hui Wang - The author contributed to the conceptualization and participated in writing and editing the review. 